\title{
L'usage des noms d'animaux pour l'éloge et le blâme en français et en arabe égyptien
}

\author{
Par \\ Tarek Abdel-Naïm Assem \\ Maître de conférences-Faculté Al-Alsun (des Langues) \\ Université de Sohag
}

DOI: $10.21608 /$ jpsu.2021.52135.1028 


\section{Le résumé de la recherche}

Cette recherche vise à éclairer les noms d'animaux utilisés pour l'éloge et le blâme en français et en arabe égyptien. Cette étude intéresse les chercheurs dans le domaine de la sociolinguistique et de la linguistique en général.

La problématique de ce travail consiste à mettre l'accent sur les points de convergence et ceux de divergence dans les deux langues. Nous tentons aussi de répondre à la question primordiale suivante : quelle est la valeur sémantique et pragmatique de l'usage de tels termes et leur influence sur les destinataires?

Nous étudions également les deux termes en français et en arabe égyptien pour atteindre leur influence sur les autres. Nous mettons l'accent sur le destinateur lui-même et le fonctionnent à ce niveau comme des marqueurs sociaux. Leur rôle linguistique est généralement lié à des variabilités sociales concernant la personne qui parle tels que l'âge, le sexe et le milieu social.

Dans la première partie de la recherche, nous abordons l'utilisation des noms d'animaux pour l'éloge dans la société française et égyptienne. Nous mentionnons également la diversité du nombre de mots utilisés pour l'éloge et leurs connotations en français et en arabe égyptien, avec des exemples de noms d'animaux mentionnés par les Français et les Égyptiens.

Quant à la deuxième partie de la recherche, nous traitons l'utilisation des noms d'animaux pour le blâme en français et en arabe égyptien en indiquant les noms d'animaux liés au blâme et leurs connotations, cela est accompagné d'exemples de blâme mentionnés par les locuteurs dans les deux sociétés: française et égyptienne.

Mots clés : éloge, blâme, animaux, signification, société 


\section{مستخلص البحث}

يهدف هذا البحث لإلقاء الضوء على أسماء الحيوانات المستخدمة في أسلوب المدح والذم داخل المجتمع الفرنسي والمصري، وتهم هذه الدراسة الباحثين في مجال علم اللغة الاجتماعي بوجه خاص واللغويات التهاء بشكل عام.

ترتكز مشكلة البحث على تحديد نقاط التشابه والاختلاف بين اللغتين فيما يخص استعمال أسماء

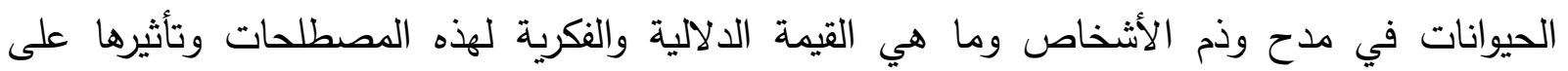

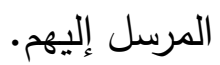

في الجزء الأول من البحث نتناول استخدام أسماء الحيوانات فى المدح داخل المجتمع الفرنسى والمصرى

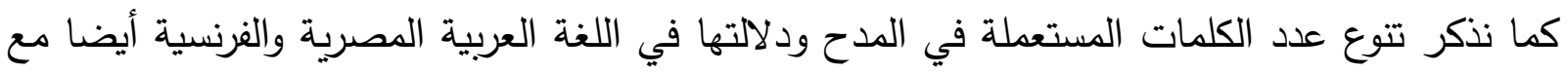
ذكر أمثلة بأسماء الحيوانات التي ورد ذكرها على لسان الفرنسيين والدصريين.

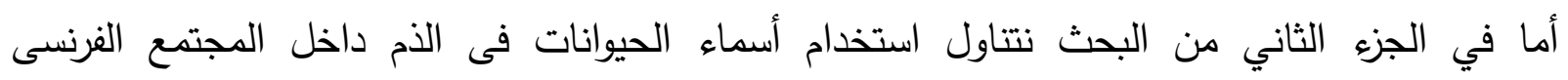
والمصرى مع ذكر أسماء الحيوانات المتعلقة بالذم ودلالتها مصحوبة بأمثلة في الذم في اللغة العربية

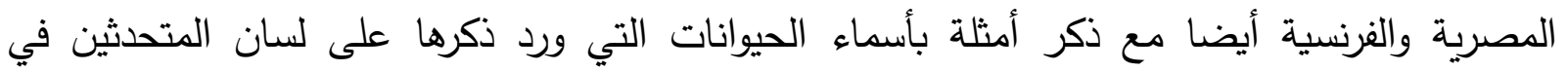
المجتمع المصري والفرنسي. كلمات مفتاحية : مدح ، ذم ، حيوانات، دلالة، مجتمع 
Il est bien remarquable que les études consacrées aux termes d'éloge ${ }^{1}$ et de blâme $^{2}$ sont rares en français et en arabe égyptien. Les termes d'éloge et ceux de blâme appartiennent à ce qu'on appelle la sociolinguistique, cette discipline qui traite la manière avec laquelle n'importe quelle société se sert des idiomes pour remplir son vouloir dire. Comme les termes en question sont employés pour exprimer des sentiments humains: éloge, approbation, critique, reproche et désapprobation, ils sont par conséquent, semblables et parfois identiques dans presque toutes les langues sans oublier pour autant les spécificités idiomatiques de chacune de ces langues. Les diverses interactions verbales entre les deux langues : l'arabe égyptien et le français, via les rapports à la fois conflictuels et amicaux, font en sorte que les termes d'éloge et les termes de blâme prennent leur chemin de la première langue à la deuxième et vice versa. Cela est bien clair lorsqu'on procède à l'étude des termes concernés dans l'arabe égyptien et leur équivalent en français.

La problématique de ce travail consiste à mettre l'accent sur les points de convergence et ceux de divergence dans les deux langues. Nous tenterons aussi de répondre à la question primordiale suivante : quelle est la valeur sémantique et pragmatique de l'usage de tels termes et leur influence sur les destinataires $?$

Nous allons étudier également les deux termes en français et en arabe égyptien pour atteindre leur influence sur les autres. Nous mettrons l'accent sur le destinateur lui-même et le fonctionnent à ce niveau comme des marqueurs sociaux. Leur rôle linguistique est généralement lié à des variabilités sociales concernant la personne qui parle tels que l'âge, le sexe et le milieu social.

Dans notre étude, les expressions d'éloge et celles de blâme sont classées en deux langues en fonction du registre de langue dont ils relèvent. Nous pouvons dire que la corrélation entre le choix de langue et les classes sociales différentes dans la société reflète le milieu social du destinateur. Si le niveau linguistique des termes employés descend vers le bas, c'est-à-dire vers le

1 - LAROUSSE Dictionnaire encyclopédique illustré définit l'éloge : paroles ou écrits qui vantent les mérites, les qualités de qqn, de qqch.

2 - LE ROBERT Dictionnaire d'Aujourd'hui définit le blâme : opinion défavorable, jugement de désapprobation (sur qqn ou qqch.) : condamnation, critique, réprobation, reproche.

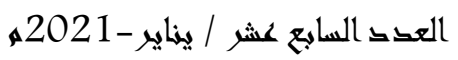


vulgaire, nous descendrons au niveau inférieur de la stratification sociale. Nous examinerons l'usage de ces termes en tant que marqueurs sociaux dans les différents milieux sociaux dans les deux langues. Nous allons passer en revue les expressions d'éloge et de blâme les plus fréquentes en nous basant sur les champs lexicaux les plus courants dans les deux langues.

Jean de la Fontaine se sert d'animaux pour instruire les hommes. Grâce au caractère qu'il attribue à chaque animal, il laisse deviner qu'il met en cause. Le lion représente le pouvoir du roi, le chat représente l'hypocrite, le renard représente le rusé.

Les termes d'éloge possèdent la même place et la même importance dans cette étude, pour cela nous allons traiter et analyser quelques termes dans les deux langues en montrant leur fonction communicative.

\section{Variété des mots d'éloge}

Le champ lexical des termes d'éloge est également plus vaste, plus riche et plus utilisé en arabe égyptien et en français courant pour s'adresser aux proches, aux amis, aux membres de la famille, aux amoureux et même aux enfants. Les mots d'éloge sont omniprésents dans toutes les interactions verbales de chaque peuple et chaque locuteur exprime de sa manière les termes assortis qu'il choisit pour attirer l'attention de son partenaire ou manipuler les autres. Les auteurs du Dictionnaire de linguistique et des sciences du langage (1994:20) donnent un sens au mot affectif : "on appelle langage affectif ou expressif celui qui traduit l'intérêt personnel que nous prenons à nos paroles par une manifestation naturelle et spontanée des formes subjectives de la pensée». Nous allons passer en revue les termes essentiels qui remplissent les fonctions pragmatiques de cette catégorie sociolinguistique.

Le nombre des termes d'éloge en arabe égyptien est très grand et laisse la liberté à chaque individu de créer des mots qui se construisent immédiatement pendant l'opération communicationnelle et en fonction de l'état moral de l'interlocuteur et la satisfaction complète de l'allocutaire en recevant la parole. Le choix des mots, dans ce cas, est donc réservé à la créativité individuelle. Le registre de cette sorte des mots contient beaucoup d'exemples et beaucoup de situations vivantes.

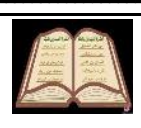

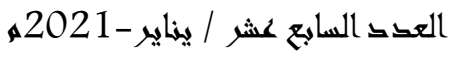


Nous commençons par les termes que les jeunes utilisent et qui occupent une priorité très grande dans la société égyptienne car ces jeunes sont les premiers à les utiliser pour exprimer leur admiration aux femmes. Voici quelques exemples:

-Ya Ghazale !, l'équivalent en français est: Tu es gazelle !

C'est une phrase adressée par un jeune homme à une belle femme afin qu'il lui plaise pour montrer sa beauté.

-Di Faraça !, l'équivalent en français est: Elle est jument !

C'est une phrase adressée par un jeune dragueur à une femme qui a un bon physique pour exprimer la féminité. La jument est la femelle du cheval, c'est une connotation indirecte pour que l'homme exprime son amour envers cette femme qui possède des caractères corporels impeccables.

Dans les deux exemples précédents, les deux termes d'éloge (Ghazale/Faraça) sont employés pour exprimer les grâces de la femme. Mais parfois ces mots dérangent les femmes parce qu'ils portent des connotations sexuelles et la loi égyptienne punit tous ceux qui profèrent ces mots.

- Al-Ahli Assad Afriquia ! l'équivalent en français est: Al-Ahli est le lion d'Afrique !

Dans le milieu social sportif, ce slogan symbolise la force de cette équipe dominant la plupart des championnats. Les journalistes et les présentateurs des programmes sportifs à la télévision utilisent le mot "lion" pour suggérer la suprématie, la force et la majesté. Les supporters de cette équipe utilisent ce slogan pour faire peur les adversaires.

- Zéab El-Gabal ! l'équivalent en français est : Les loups de la montagne !

Cette expression est attribuée à une équipe égyptienne appelée «AlMoqawloun Al-Arab» qui participe dans le tournoi égyptien. Cette nomination signale que les loups sont ici les maîtres de la montagne.

- Di Yamama! l'équivalent en français est : Elle est tourterelle ! 
La tourterelle représente la beauté de la femme et ses grâces.

- Di Nahla! l'équivalent en français est : Elle est comme l'abeille!

L'abeille est le symbole du travail et de la persévérance. La personne qui travaille beaucoup et qui est actif, on l'appelle "Nahla".

En français, les termes d'éloge existent partout dans les différents milieux sociaux. Mais avant de citer quelques exemples, nous pouvons montrer la définition de ce mot. L'éloge, c'est un discours à la louange de quelqu'un ou de quelque chose. Le registre français est riche de ces vocabulaires.

Le tableau suivant montre l'usage sémantique de quelques animaux pour présenter des qualités aux hommes en ce qui concerne l'éloge.

\begin{tabular}{|l|l|l|}
\hline \multicolumn{1}{|c|}{ Animal } & \multicolumn{1}{|c|}{ Signification } & \multicolumn{1}{c|}{ Exemple } \\
\hline Le lynx & La perception & Il a des yeux de lynx. \\
\hline Le putois & La confiance en soi & Je crie comme un putois. \\
\hline L'abeille & Le travail & $\begin{array}{l}\text { Il est travailleur, il travaille } \\
\text { comme l'abeille. }\end{array}$ \\
\hline L'agneau & L'innocence & $\begin{array}{l}\text { Cet enfant est innocent } \\
\text { comme l'agneau. }\end{array}$ \\
\hline Le cheval & La puissance & $\begin{array}{l}\text { Sa force est de 12 000 } \\
\text { chevaux. }\end{array}$ \\
\hline Le chevreuil & La douceur & $\begin{array}{l}\text { Je le trouve doux comme le } \\
\text { chevreuil. }\end{array}$ \\
\hline Le chien & La fidélité & Il est fidèle comme le chien. \\
\hline La tortue & La majesté & Vous êtes le lion ici. \\
\hline
\end{tabular}


Au sens péjoratif, le cheval est un synonyme parfois utilisé pour désigner une personne.

- Elle est tombée sur un bon cheval.

En France, le corbeau représente l'intelligence mais en Égypte il représente la laideur et le pessimisme tandis que la colombe représente la beauté.

Signalons aussi que les connotations de certains de ces termes d'éloge contiennent des mots qui appartiennent aux champs sémantiques de la beauté comme «Enti gazala!»» « littéralement, Tu es gazelle!». Cela signifie que cette femme est mince et sa beauté est attirante.

Rappelons que l'usage de ces termes d'éloge dépend de la personnalité de l'interlocuteur et son choix des mots et à qui il adresse sa parole. La nature de son acte de parole est parfois une sorte de flatterie, de courtoisie ou de politesse.

L'arabe égyptien et le français sont donc deux langues qui comportent beaucoup de termes d'éloge et leur objectif est d'encourager le destinataire de façon à le rendre gai de ses compétences, de ses performances ou de ses capacités. Les termes d'éloge jouent un rôle très important dans la communication parce qu'ils permettent aux locuteurs d'inventer leurs termes et d'exprimer librement leurs sentiments à leurs partenaires. Pragmatiquement, les termes d'éloge laissent la porte ouverte devant les néologismes ${ }^{1}$ qui envahissent les deux langues surtout l'arabe égyptien à travers le nombre de mots que les interlocuteurs empruntent à d'autres situations qu'ils ont vécues ou vues au cinéma et à la télévision. Le portable est maintenant un moyen efficace aux allocutaires de connaitre une ou plusieurs langues étrangères et comprendre l'usage de nouveaux termes dans ce domaine.

Pour terminer cette partie, nous pouvons dire que chaque peuple possède son répertoire de termes d'éloge que seuls les natifs comprennent par cœur leur emploi et leur signification.

\footnotetext{
1 - Le néologisme est un mot nouveau ou récemment forgé pour répondre à un manque ou pour son caractère expressif.
} 


\section{L'emploi des noms d'animaux pour le blâme en français et en arabe égyptien.}

\section{Termes d'injures dans l'interaction quotidienne}

Les injures sont très courantes dans la langue parlée entre les différentes personnes, c'est-à-dire qu'elles sont très fréquentes dans les interactions quotidiennes ainsi que dans les différentes situations de la vie. Elles sont très employées dans les moments de vraie colère où les personnes expriment leur agacement, leur dégout ou même leur indifférence les uns avec les autres. D'autre part, nous avons des termes injurieux qui sont utilisés entre les amoureux à des fins humoristiques ou par plaisanterie. Dans les milieux égyptiens et ceux de français, ces termes ne représentent pas une menace pour la face du destinataire, ni pour celui qui les profère.

Les expressions injurieuses utilisées dans leur vrai sens sont toujours précédés de la particule vocative [ya] qui donnent l'équivalent français de " espèce de...». En d'autres termes, ces mots sont classés suivant leur place de vulgarité appelée par Parkinson «the heaviness scale». Voici quelques termes :

- Di Akraba !, l'équivalent en français est: Espèce de scorpion!

- Di Hashara ! , l'équivalent en français est: Espèce d'insecte !

-Ya homar !, l'équivalent en français est: Espèce d'âne !

-Ya Kalb !, l'équivalent en français est: Espèce de chien !

-Ya Khannzeir !, l'équivalent en français est: Espèce de cochon !

- Ya Ghorab !, l'équivalent en français est: Espèce de corbeau !

- Enti Bouma !, l'équivalent en français est: Espèce de hibou !

- Di Hérbaya !, l'équivalent en français est: Espèce de caméléon !

- Da Sorsar !, l'équivalent en français est: Espèce de cafard. ! 
Chaque injure est fortement liée à la classe sociale de celui qui tient la responsabilité de la parole. Rappelons que les personnes de la classe populaire ne trouvent aucune pudeur à utiliser les expressions interdites en parlant avec les membres de la même classe. Contrairement à ce milieu inferieur, les classes supérieures ou moyennes refusent complètement de proférer ces termes blessants. Le choix de ces termes est lié de premier ordre au degré de colère du locuteur et son interaction au moment de l'énonciation. En règle générale dans les deux langues de notre corpus, plus nous sommes en colère ou un moment de rage, plus nous descendons au niveau inférieur de l'échelle des expressions injurieuses. Les personnes qui représentent la classe moyenne peuvent utiliser ces termes lourds lorsqu'elles sont en colère ou excitées dans une situation difficile. Notons également que le niveau personnel de politesse joue un rôle très important dans l'usage de ces expressions jugées tabous dans les normes sociales de chaque peuple. Une minorité de la classe moyenne utilise ces expressions blessantes, tandis que la majorité française et égyptienne appartenant à cette classe refuse catégoriquement d'employer ces injures quel que soit leur niveau de vulgarité et n'utilise que quelques mots dits «légers» dans le cas de l'extrême colère. Par contre, pour beaucoup de personnes qui appartiennent à la classe populaire, l'emploi de ces injures est une partie essentielle de leurs habitudes quotidiennes et ils les utilisent avec leurs proches même avec les petits !

Il faut rappeler que ces expressions peuvent être utilisées entre les personnes inconnues dans les situations variées de la vie quotidienne en cas de colère aussi bien entre les amis et les camarades pour plaisanter ou pour exprimer leur réaction contre ce qui blesse les sentiments. Voici deux exemples :

-Enta Homar !, c'est-à-dire: Tu es âne !

-Enti Baqara!, c'est-à-dire : Tu es vache !

Nous pouvons dire que ces termes sont utilisés entre les intimes pour rigoler, mais d'une manière sérieuse, ils sont employés dans les différentes situations de la vie pour exprimer les réactions négatives en face de quelqu'un ou quelque chose qui causent directement des problèmes. Ces termes accroissent dans les milieux populaires pauvres et parmi les familles besogneuses. 


\section{Classification lexicale des expressions de blâme}

Malgré la grande différence entre les termes de blâme en arabe égyptien et en français, les deux langues ont beaucoup de termes dans ce domaine. Les français publient des livres et des dictionnaires dans lesquels il y a plusieurs mots de blâme qui relèvent des niveaux dits : légers, moyens, lourds. Les égyptiens ont également un grand nombre des injures et des insultes qui dépasse dix mille d'après les dernières statistiques. Pour les deux langues, les champs lexicaux sont vastes et embrassent plusieurs sortes d'injures.

En parcourant le champ lexical dont relève une grande quantité des expressions injurieuses est pratiquement celui des noms d'animaux soit sauvages ou domestiques. Cette catégorie d'injure est différente d'un pays à l'autre, il y a des peuples qui acceptent d'une manière satisfaisante les appellations des noms d'animaux et par contre, il y en a qui refusent totalement cette appellation qui sont pour eux des insultes directes condamnées par la loi.

\section{Les termes courants d'injure}

Il faut noter que le choix de ces termes dans les deux langues est bien sélectionné et son objectif est d'agacer et déranger les destinataires. Chaque nom d'animal contient quelque chose de péjoratif vis-à-vis du destinataire et sa manière de parler ou de raisonner. Le but essentiel en prononçant ces gros mots est de montrer les défauts des personnes concernées. Le mot clé ou générique de ces champs lexicaux est (Hayawan/hayawana) en arabe égyptien, la même chose et la même influence en français viennent de mot (bête) pour le masculin et le féminin. Nous pouvons avec beaucoup de facilité trouver ces deux termes dans la vie quotidienne en France et en Egypte. Voici quelques échantillons :

-Enta Hayawan! Ou Enty Hayawana! En français : Tu es bête!

Nous pouvons dire en français : C'est une question bête! C'est-à-dire

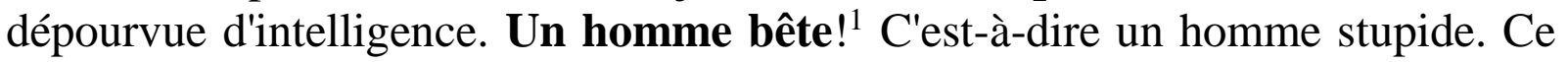
mot, en français, est toujours adjectif mais il porte le synonyme d'un nom. Nous

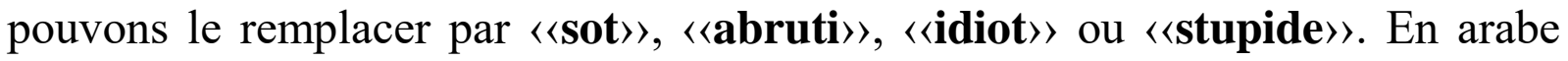
égyptien, son sémantisme est le même qu'en français mais nous ajoutons qu'il 
signifie : il ne comprend rien ou il est ignorant, c'est-à-dire qu'il n'a aucun savoir. Dans les deux langues, le destinataire ne se comporte pas comme un être humain mais comme un animal. Les animaux qui s'emploient beaucoup dans ce domaine en arabe égyptien sont (Homar/Homara) Homar pour le masculin et Homara pour le féminin. En français, le mot (âne m. /f. ou ânesse) est un terme qui montre également la stupidité flagrante du destinataire. Voilà des exemples:

-Enta Homar Mabtifhmsh! (Tu es âne! Tu ne comprends pas !)

-El Homara ely Bititkalem ! (L'ânesse qui parle !)

Un professeur en s'adressant à une étudiante qui fait du bruit ou bavarde dans la classe. Dans l'arabe égyptien, l'âne joue un rôle essentiel quand nous voulons humilier quelqu'un et l'obliger à respecter la loi ou les règles suivies dans la société. En français moderne comme l'ancien français, l'âne est également le symbole de l'ignorance et la stupidité. Il est polysémique et son rôle est varié. L'expression la plus courante dans les milieux populaires français est (être comme l'âne), c'est-à-dire qu'il est idiot et ignorant comme l'âne. Il est l'équivalent des mots suivants : bourrique, bête, baudet, mule, mulet. Nous pouvons entendre facilement ces termes en lançant des injures.

-Il est mule !

-Tu es bourrique, toi ! C'est-à-dire une personne sotte et entêtée.

Ce terme est aussi employé pour manifester un mépris et une colère à un imbécile. Dans ce cas, l'âne est le synonyme des adjectifs suivants : sot, imbécile, ignorant, buse, crétin, stupide, etc.

Il est âne. ----- Il est sot.

Il est âne. -----Il est imbécile.

Il est âne. -----Il est ignorant.

Les proverbes et les expressions français utilisent le mot (âne) pour les mêmes finalités, c'est-à-dire qu'on qualifie quelqu'un d'âne pour designer qu'il est hébété et n'arrive à bien raisonner. Voici quelques proverbes montrant les mêmes connotations :

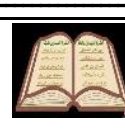


-L'âne frotte l'âne. C'est-à-dire que les imbéciles s'encouragent entre eux.

-âne bâté. Qui ignore beaucoup de choses par stupidité.

-Oreilles d'âne. Les longues oreilles symbolisent la pesanteur d'esprit.

En arabe égyptien, le petit de l'âne est nommé (Gahsh), c'est-à-dire (ânon) en français, ce terme est fréquemment utilisé pour montrer le caractère têtu et peu intelligence de la personne. Il est beaucoup plus blessant que le mot (Homar) et son équivalent dans la vie quotidienne égyptienne est (Teiss), (bouc). Les professeurs qualifient les étudiants qui ne comprennent pas leur leçon et restent la bouche bée par le mot (Teiss). Voici un exemple :

-El walad da Teiss Kebeir ! C'est-à-dire qu'il est un grand bouc.

Pour le reste des noms d'animaux, les lexiques égyptiens des injures sont plus riches que le registre français de cette catégorie. Nous pouvons citer quelques injures courantes dans la rue égyptienne.

-Ibn elKalb! (le fils du chien)

-Bent elkalb! (la fille du chien)

Ces deux injures marquent la bassesse et la vilenie. Les mots (Kalb et kalba) qui impliquent les mêmes connotations sont habituels en Egypte surtout entre les jeunes garçons et les jeunes filles mais la loi punit les interlocuteurs qui emploient ce gros mot dans les places publiques. Par plaisanterie, on peut utiliser ces termes entre les amis et les proches. Voici un exemple :

-El Kalb da mosh byzakr drossoh! Un père qui adresse la parole à son fils qui n'étudie pas ses leçons. Il dit : «ce chien n'étudie pas ses leçons!»»

-Elkalba di wahshani! Une jeune fille dit à son amie en plaisantant : «cette chienne me manque beaucoup!»»

Contrairement à l'arabe égyptien, le terme «chien» en français n'est pas une insulte mais il est le symbole de la fidélité et l'amitié. Si on dit à quelqu'un : Tu es fidèle comme le chien, il va répondre en disant : Merci monsieur, c'est la 
gentillesse de votre part! Il représente donc des qualités comme l'honnêteté et la loyauté.

Nous avons un autre terme d'injure animale qui joue un rôle important dans les deux langues, c'est le mot «khanzeir» en arabe égyptien et «(cochon») en français. Dans la société française, le cochon est un gros mot et la loi punit les personnes qui l'utilisent. Si on dit: sale cochon! Le destinataire qui reçoit ce terme réagit vite et d'une manière agressive contre l'interlocuteur. La loi française interdit son usage dans la presse écrite et électronique ainsi que dans la rue et les lieux publics. Le chanteur belge Jacques Brel a insulté les bourgeois en utilisant le terme «cochon») dans sa chanson, il dit: « Les bourgeois, c'est comme les cochons, plus ça devient vieux, plus ça devient bête! $\rangle^{1}$

Nous avons également vu, par hasard, en lisant le journal électronique «Midi-Libre» un commentaire d'une lectrice française Christine Tasin le 19 février 2013, elle a dit: 〈et si à la place du cheval, ça avait été du cochon? »). Son commentaire est resté un quart d'heure sur le site avant d'être censuré. Elle a montré que les responsables du journal électronique ont répondu :

« Bonjour, certains de vos commentaires sont contraires à notre charte qui proscrit notamment les propos injurieux, racistes, diffamatoires, agressifs.)>

Le mot cochon marque aussi en français la saleté et l'indifférence totale. En arabe égyptien, nous avons la même fonction et la même influence.

Le tableau suivant indique l'usage de quelques animaux pour présenter des inconvénients aux hommes. Au dix-septième siècle, La Fontaine, dans ses fables, a excellé à révéler les défauts des personnes et leurs comportements au moyen des animaux comme le lion, le corbeau, la colombe, la fourmi, le renard...etc. 


\begin{tabular}{|l|l|l|}
\hline \multicolumn{1}{|c|}{ Animal } & \multicolumn{1}{|c|}{ Signification } & \multicolumn{1}{c|}{ Exemple } \\
\hline Le renard & La ruse & Ce voleur semble un renard. \\
\hline L'abeille & La méchanceté & Elle pique comme l'abeille. \\
\hline L'âne & L'ignorance & Il ne sera jamais qu'un âne. \\
\hline Le chat & Le mal & $\begin{array}{l}\text { Elle griffonne comme la } \\
\text { chatte. }\end{array}$ \\
\hline Le lapin & La peur & $\begin{array}{l}\text { Cet enfant agit comme le } \\
\text { lapin. }\end{array}$ \\
\hline
\end{tabular}

Spécifiquement, l'abeille est la représentation symbolique du pouvoir et du règne de Napoléon :

- Envolons-nous, comme les abeilles du manteau impérial devant les exhortations hugoliennes!

Mais il faut signaler que certains animaux donnent deux significations contrariées :

L'abeille, par exemple, symbole du travail et à la fois de la méchanceté. Pour le chat, en Égypte antique, cet animal est le symbole de la force, l'aide à l'homme, et l'agilité. Mais en France, selon les régions, il est le symbole de la malchance et le mal et sinon la protection contre le mauvais œil.

Le chien, aussi, symbole de la fidélité chez les Français et les Égyptiens. Mais quelques Égyptiens disent "Enta klab", c'est-à-dire "Tu es chien" pour insulter quelqu'un. Au niveau familier, au sens péjoratif, le lapin est attribué à la personne qui a beaucoup d'enfants.

- Les voisins sont de vrais lapins, ils ont sept enfants. 


\section{Est-ce que vous entendez des expressions de blâme ?}

Oui bien sûr. D'abord, il faut comprendre la langue. Beaucoup disent des choses horribles dans une langue qu'on ne comprend pas. Il y a les insultes de base, les classiques. Il y a les insultes à soi-même. Si on est sur un court couvert par la télé et que tout le monde entend l'insulte. Vous êtes moins flexible que si c'est sur une courte annexe où on peut considérer ça comme une discussion privée d'hommes. Entre nous. Ici, il y a des micros tous les mètres. Plus il y a la télévision plus les joueurs font attention à leur image. Si un joueur dit un «putain» dans les bâches et qu'à la télévision on n'entend que ça, le téléspectateur ne comprendrait pas forcement que le joueur dise cela et que l'arbitre reste impassible.

Enfin, nous citons quelques termes qui possèdent des sens injurieux et qui sont fortement utilisés en Egypte. Tel est le cas de l'expression (lama yegy ebn el kalb habaho!) (Littéralement Fils du chien ! Quand il viendra, je l'abattrai !) qui a trois connotations : la menace, l'injure et la malédiction. Les parentes utilisent beaucoup cette expression pour montrer leur colère contre les enfants qui sont en train de commettre une faute quelconque, pour les corriger. Mais pour les personnes égales lorsque quelqu'un fait tort à quelqu'un d'autre, l'agressé invoque la malédiction à l'agresseur en disant cette expression. 


\section{Conclusion}

Pour conclure, nous pouvons dire que les expressions d'éloge et celles de blâme constituent un très vaste registre dans toutes les langues dont le français et l'arabe. Cela fait partie intégrante de la sociolinguistique qui s'intéresse à étudier les interactions énonciatives des personnes. De tels termes et expressions ne manquent pas du tout de la rhétorique et des figures de style. On y trouve évidemment la personnification des choses, la métaphore, la métonymie et audessus de toute la comparaison. Ce sujet pourrait subir une étude élargie d'un point de vue à la fois lexicologique, étymologique et sémantique. 


\section{Bibliographie}

-BAYLON Christian, Mignot Xavier, "La Communication", Nathan, Paris, 1991. -DOMINICY Marc et FREDERIC Madeleine, "La mise en scène des valeurs. La rhétorique de l'éloge et du blâme", Delachaux et Niestlé, Lonay, 2001.

-GOFFMAN Erving, "Les rites d'interaction", Collection "Le sens commun", Minuit, Paris, 1974.

-GUIRAUD Pierre, "L'argot", PUF, Paris, 1969.

-HERMANS Jean-Claude, "Le chien dans les fables", Société des écrivains, Paris, 2011.

-HUSTON Nancy, "Dire et interdire: éléments de jurologie", Payot, Paris, 1980. -PERGNIER Maurice, "Le Mot", Collection "Linguistique nouvelle", PUF, Paris, 1986.

-ROUAYRENC Catherine, "Les Gros mots", Collection "Que sais-je?", PUF, Paris, 1998.

-VION Robert, "La communication verbale: analyse des Interactions", Hachette, Paris, 1992.

\section{-Dictionnaires français:}

- CARADEC François, POUY Jean-Bernard, "Dictionnaire du français argotique et populaire", Larousse, Paris, 1998.

- Collectif "Dictionnaire encyclopédique illustré", LAROUSSE, Paris, 1998.

- DUBOIS Jean, "Dictionnaire de linguistique et des sciences du langage", Larousse, Paris, 1994.

- EDUARD Robert, "Dictionnaire des injures", Paris, Tchou, 2004.

- LEMONIER Marc, "Insultes, gros mots et injures", petit dictionnaire des insultes de A à Z, Mini poche. City Éditions, Hachette, Paris, 2009.

- REY Alain, "LE ROBERT Dictionnaire d'Aujourd'hui", Dictionnaires LE ROBERT, Paris, 1991.

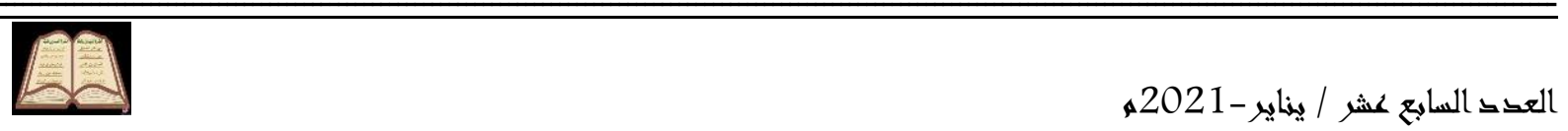




\section{-Sites électroniques}

-www.superprof.fr, consulté le 21/8/2020, $10 \mathrm{~h}$.

-www.academia.edu, consulté le 11/9/2020, $15 \mathrm{~h}$.

-www.paroles.net, consulté le 11/9/2020, 16 h.

-www.20minutes.fr, consulté le 4/10/2020, 20 h.

-www.dictionnaire.sensagent.leparisien.fr/dimunitif/fr-fr/, consulté le

$15 / 10 / 2020,23 \mathrm{~h}$.

- www.rts.ch/info/culture/livres, consulté le 17/10/2020, 10 h.

\section{قواميس مصرية عن المدح والذم}

-أحمد أمين: قاموس العادات والتقاليد والتعابير المصرية, كلمات عربية للترجمة والنشر , 2013. -عمرو المنشاوي: قاموس الثتائم المصرية, إبداع للترجمة والنشر والتوزيع, 2017. 